\title{
Rho Production with Linearly Polarized Photons
}

\author{
L. Criegee, G. Franke, G. Löffler, K. P. Schüler, U. Timm, and W. Zimmermann \\ Deutches Elektronen-Synchrotron, Hamburg, Germany \\ and \\ H. Werner \\ Max-Planck-Institut für Kemphysik, Heidelberg, Germany \\ and \\ P. W. Dougan \\ University of Lund, Lund, Sweden \\ (Received 16 June 1970)
}

The process $\gamma p \rightarrow \rho^{0} p \rightarrow \pi^{+} \pi^{-} p$ has been studied with linearly polarized photons between 1.4 and $3.3 \mathrm{GeV}$. We observe almost pure diffractionlike behavior in the whole energy region.

We have measured the correlation between photon polarization and $\rho$ decay for the reaction

$$
\gamma \rho \rightarrow p \rho^{0}-p \pi^{+} \pi^{-}
$$

in the photon energy range $1.4 \leqslant k \leqslant 3.3 \mathrm{GeV}$ in order to check the predictions of different production models. The decay $\rho^{0} \rightarrow 2 \pi$ was observed for decay planes near a direction $\overrightarrow{\mathrm{n}}_{H}$, normal to the horizontal symmetry plane of the detection system, such that we obtained the counting rates $Y_{\text {}}$ when the linear photon polarization was directed parallel to $\overrightarrow{\mathrm{n}}_{H}$, and $Y_{\perp}$ for an orientation perpendicular to it. These yields are combined to form the asymmetry ratio

$$
\frac{1}{P_{\gamma}} \frac{Y_{\|}-Y_{\perp}}{Y_{\|}+Y_{\perp}}=\frac{\sigma_{\|}-\sigma_{\perp}}{\sigma_{\|}+\sigma_{\perp}}=\Sigma,
$$

where $P_{\gamma}$ is the degree of polarization, and $\sigma_{\|}$ and $\sigma_{\perp}$ are the cross sections for Reaction (1) from photons with electric vector parallel and perpendicular, respectively, to $\overrightarrow{\mathrm{n}}_{H}$. $\Sigma$ is indicative of the $\rho$ production mechanism: In the limit of high energies, natural exchange of parity leads to $\Sigma=+1$; unnatural parity exchange yields $\Sigma=-1 .^{1}$ Independent of energy, the diffraction model and the one-pion-exchange (OPE) model predict $\Sigma=+1, \quad \sigma_{\perp}=0$ and $\Sigma=-1, \sigma_{\|}=0$, respectively.

The experimental setup is similar to the one used in an earlier experiment. ${ }^{2}$ A well-collimated and linearly polarized photon beam produced by coherent bremsstrahlung from a diamond target traversed a liquid hydrogen target. Each of the three final-state particles of Reaction (1) was detected in its own counter-wire plane telescope, the two identical pion telescopes being mounted symmetrically above and below the plane defined by the axis of the proton telescope and the beam. In addition to all angles, range and $d E / d x$ of the proton were measured. The detection efficiency of the proton telescope was improved compared with the earlier setup by using $X-Y$ readout in four of the wire planes. In order to cover the photon energy range from 1.4 to $3.3 \mathrm{GeV}$ several settings of the diamond spectra and of the telescope were used. Spectra were monitored simultaneously by a pair spectrometer. The beam polarization $P_{\gamma}$ was obtained by applying standard fitting procedures to the measured spectra. ${ }^{3}$ After a three-telescope coincidence, wire plane and ADC information was read into a small computer and transferred onto magnetic tape. The analysis was done off line. We evaluated 188000 events which in each telescope had one track inside a chosen fiducial volume. The rate of three-track events per monitor unit was constant throughout the experiment within statistical errors. For each event the photon energy $k$ was calculated in two different ways, using and not using energy conservation. The difference, $\Delta k$, between the two is zero for elastic $2 \pi$ events within the experimental resolution. Plots of the frequency of events versus $\Delta k$, as shown in Fig. 1, were used to subtract the inelastic background from $p \pi \pi$ events concentrated near $\Delta k=0$.

The asymmetry ratio $\Sigma$ was calculated from events whose di-pions were distributed over a finite solid angle of $\Delta \Omega=0.64 \mathrm{sr}$ around the parallel orientation of polarization, in the $\rho$ rest frame. A check with $\Delta \Omega=0.16 \mathrm{sr}$ yielded statistically the same result for $\Sigma$. To account for the variation of cross sections over this range we used the general decay distribution of $\rho$ mesons 


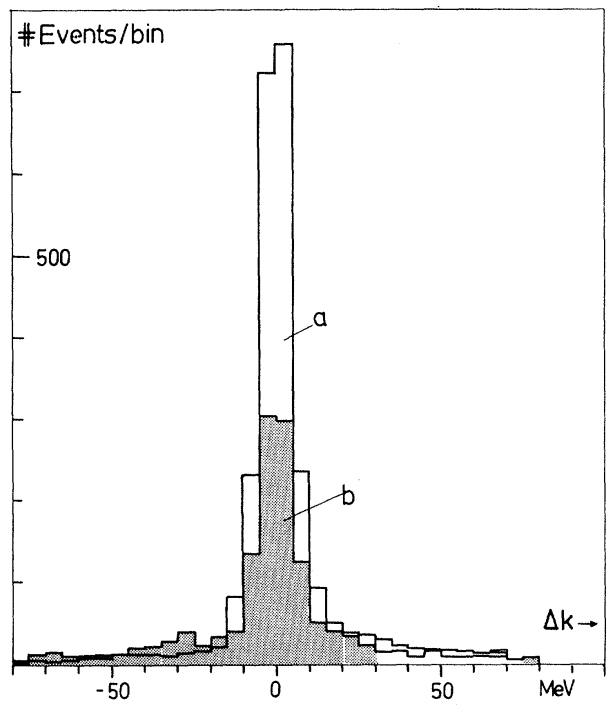

FIG. 1. Number of events versus kinematical constraint variable $\Delta k$ for two different ranges of proton kinetic energy $T_{p} . p \pi \pi$ events are concentrated near $\Delta k=0 . a, 70 \leqslant T_{p} \leqslant 97 \mathrm{MeV} ; b, 145 \leqslant T_{p} \leqslant 172 \mathrm{MeV}$.

from polarized photons, as given by Schilling, Seyboth, and Wolf, ${ }^{1}$ and specialized it to the two predictions for diffraction $(+)$ and OPE (-):

$$
W^{ \pm}(\theta, \psi) \sim \sin ^{2} \theta\left(1 \pm P_{\gamma} \cos 2 \psi\right),
$$

where $\theta$ is the polar angle and $\psi$ the azimuthal angle of one of the pions with respect to the beam polarization. The solid-angle cut restricts $\psi$ to the two ranges $\psi=0 \pm 0.4$ and $\psi=\frac{1}{2} \pi \pm 0.4 \mathrm{rad}$ for parallel and perpendicular orientation, respectively. Thus we assume for our experimental yields the distribution

$$
Y \sim \sigma_{\|} W^{+}(\theta, \psi)+\sigma_{\perp} W^{-}(\theta, \psi) .
$$

The choice of polar axis for the $\rho$ rest frame, which is specific to the two models, turns out to be of minor importance in our case, since we observe pions around the normal to the production plane, $\theta \approx \frac{1}{2} \pi$. We have chosen the direction of the incoming proton as polar axis. Apart from the solid angle, we applied the following further restrictions to the events admitted for analysis:

(1) The reconstructed photon energy had to be in a region of high polarization below the spike of the coherent spectra. ${ }^{3}$

(2) The di-pion mass was restricted to the region of the $\rho$ resonance, $0.65<m_{\pi \pi}<0.90 \mathrm{GeV} / c^{2}$. Nonresonant di-pions were not subtracted. Fig- ure 2, as an example, shows that this background is low.

(3) The constraint measure $|\Delta k|$ had to be smaller than $20 \mathrm{MeV}$. The number of inelastic

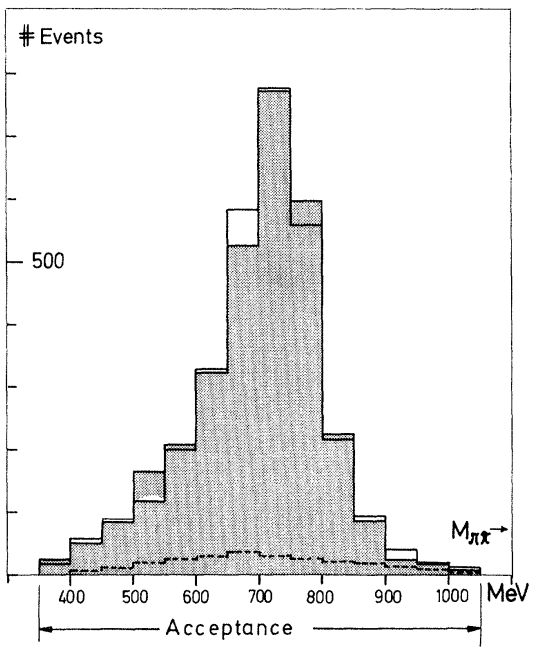

FIG. 2. Comparison of the observed $\pi \pi$-mass spectrum with the result given by a Monte Carlo calculation. The parameters used for the $\rho^{0}$ shape are $M_{0}$ $=755, \Gamma_{0}=140 \mathrm{MeV}$. Shaded area, distribution of observed events; solid line, distribution of fake events including $8 \%$ phase space; dashed line, phase-space distribution.

events inside this region was computed from outside bins and subtracted.

By these criteria $13000+18000$ events were selected for the two polarizations. Data from the previous experiment ${ }^{2}$ are included in these numbers.

The errors of $\Sigma$ include statistical and systematic ones, where the latter are due to the following sources: (1) the calculated polarization in the coherent spike, ${ }^{3}$ for which we allow an error of $\Delta P_{\gamma} / P_{\gamma}=0.05$, which propagates into $\Sigma$; (2) uncertainty in the reconstructed photon energy producing a $5 \%$ error in $\Sigma$; (3) inelastic proton scattering which reduces the range of the protons, demonstrated by Monte Carlo calculations to have negligible effect on $\Sigma(<1 \%)$. These errors have been added quadratically to the statistical errors. Figure 3 shows $\Sigma$ as a function of momentum transfer for three different photon energy ranges. The solid line was calculated using published cross sections for diffractive $\rho$ production $\left[d \sigma / d t=140 \exp (+5.5 t) \mu \mathrm{b} \mathrm{GeV}{ }^{-2}\right]^{4}$ and plausible assumptions for the OPE process $\left[g_{\pi_{N N}}{ }^{2}\right.$ $\left.=14.7, \Gamma_{\rho \pi \gamma}=0.1 \mathrm{MeV}\right]$. The dashed line includes an estimate of initial- and final-state corrections to the OPE cross section, as calculated by Schilling and Storim ${ }^{5}$ for $\omega$ photoproduction. Taking $\omega p$ and $\rho^{0} p$ scattering to be equal, these corrections are scaled down to $\rho^{0}$ photoproduction by the same factor $1 / 9$ as the cross sections. 


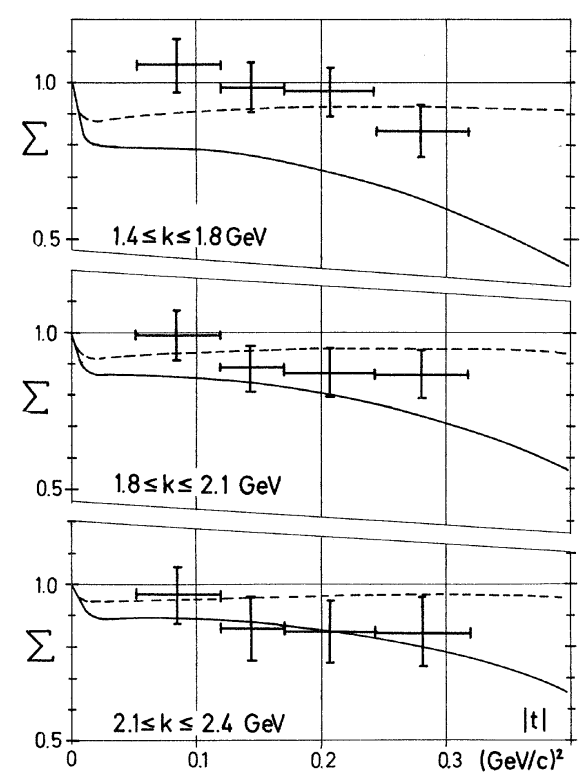

FIG. 3. Polarization asymmetry $\Sigma$ vs $|t|$ for three ranges of incident photon energies. Solid line, calculated asymmetry taking diffraction and OPE parameters into account (see text); dashed line, using the same parameter by including final- and initial-state interaction.

The data show general consistency with these calculations, and do not require larger nondiffractive contributions. The errors are not small enough, however, to allow a unique-determination of $\Gamma_{\rho \pi \gamma}$. We do not observe any influence of $s$-channel resonances decaying into $\rho+p$. This is in agreement with calculations by Schildknecht and Schrempp, ${ }^{6}$ who predict a change of $\Sigma$ smaller than $5 \%$ for $\Delta(1950)$ and smaller than $2 \%$ for $\Delta(2420)$, as long as they are coupled by magnetic

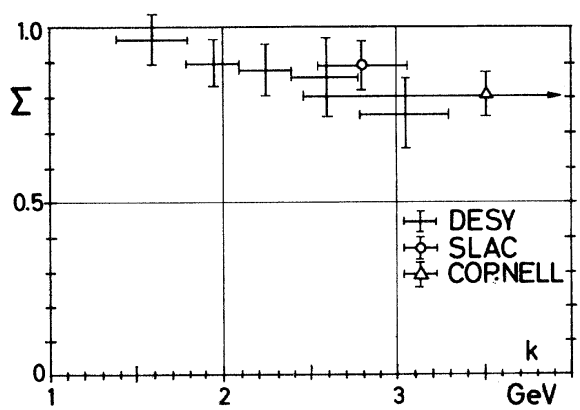

FIG. 4. Polarization asymmetry $\Sigma$ vs $k$ for $|t|<0.4$ $(\mathrm{GeV} / c)^{2}$. multipole transitions only, like the $\Delta(1236)$ resonance. In Fig. 4 we present $\Sigma$ as a function of $k$, summed over $|t|$. The experimental points at 2.6 and $3.1 \mathrm{GeV}$ were not presented in Fig. 3 because of low statistics. Experimental points obtained at the Stanford Linear Accelerator Center ${ }^{7}$ and at Cornell ${ }^{8}$ in our energy region are shown for comparison. In conclusion we find almost pure diffraction production in the range $1.4 \leqslant k$ $\leqslant 3.3 \mathrm{GeV}$, consistent with a small OPE contribution of $\Gamma_{\rho \pi \gamma} \approx 0.1 \mathrm{MeV}$, and no anomalous change of $\Sigma$ as a result of intermediate resonances.

We are indebted to the synchrotron group, the Hallendienst, and the Rechenzentrum of DESY for their efficient support. We are grateful to Professor Jentschke, Professor Lohrmann, and Professor H. Joos for encouragement, support, and helpful discussions. One of us (P.W.D.) is indebted to the Statens Rad for Atomforskning, Sweden, for financial assistance.

${ }^{1}$ K. Schilling, P. Seyboth, and G. Wolf, Stanford Linear Accelerator Center Report No. SLAC-PUB683, 1969 (unpublished).

${ }^{2}$ L. Criegee, M. H. Carrell, C. Gottfried, K. Krolzig, G. Löffler, A. Saulys, K. P. Schüler, U. Timm, W. Zimmermann, H. Werner, G. B. Collins, P. W. Dougan, E. von Goeler, and R. A. Carrigan, Phys. Lett. 28B, 282 (1968).

${ }^{3}$ L. Criegee, G. Lutz, H. D. Schulz, U. Timm, and W. Zimmermann, Phys. Rev. Lett. 16, 1031 (1966).

${ }^{4}$ Achen-Berlin-Bonn-Hamburg-Heidelburg-München Collaboration, Phys. Rev. 175, 1669 (1968).

${ }^{5} \mathrm{~K}$. Schilling and F. Storim, Nucl. Phys. B7, 559 (1968).

${ }^{6}$ D. Schildknecht and B. Schrempp, DESY Report No. 70/8, 1970 (unpublished).

${ }^{7} \mathrm{~J}$. Ballam et al., in Proceedings of the Intermational Symposium on Electron and Photon Interactions at High Energies, Liverpool, England, 1969, edited by D. W. Braben (Daresbury Nuclear Physics Laboratory, Daresbury, Lancashire, England, 1970), Abstr. No. 109, and Stanford Linear Accelerator Center Report No. SLAC/PUB-728, 1970 (unpublished).

${ }^{8} \mathrm{G}$. Diambrini et al., in Proceedings of the International Symposium on Electron and Photon Interactions at High Energies, Liverpool, England, 1969, edited by D. W. Braben (Daresbury Nuclear Physics Laboratory, Daresbury, Lancashire, England, 1970), Abstr. No. 96. 